\title{
Dementia Care Apps for People with Dementia and Informal Caregivers: A Systematic Review Protocol
}

\author{
Bing Yea, ${ }^{a}$ Tuck-Voon How ${ }^{b, c}$ Charlene H. Chu ${ }^{b, d}$ Alex Mihailidis ${ }^{a, b}, c$ \\ aDepartment of Occupational Science and Occupational Therapy, University of Toronto, Toronto, ON, Canada; \\ ${ }^{\mathrm{b}}$ Toronto Rehabilitation Institute, KITE Research, University Health Network, Toronto, ON, Canada; 'Institute of \\ Biomedical Engineering, University of Toronto, Toronto, ON, Canada; 'Lawrence S. Bloomberg, Faculty of Nursing, \\ University of Toronto, Toronto, ON, Canada
}

\section{Keywords}

Dementia care apps · Dementia · Informal caregivers

\begin{abstract}
Dementia drastically impacts the quality of life (QOL) of both people living with dementia (PLwD) and their family caregivers. As dementia progresses and care needs escalate, the likelihood of institutionalization is increased, which is counter to the wishes of the majority of older adults and their family members. Dementia care apps can provide critical support and have the potential to improve the QOL of both PLwD and their family caregivers and reduce perceived caregivers' burden. However, there is a lack of understanding of the needs of both PLwD and their family caregivers related to dementia care apps. There is also a gap in understanding the privacy concerns in relation to the apps among older adults with dementia and their caregivers. As such, the main aims of this systematic review are to understand the landscape of dementia mobile apps targeting PLwD and their caregivers with respect to the features of the apps, usability testing, and the privacy and security aspects of the app from the perspective of both app developers/researchers and the end users (PLwD and family caregivers who provide care of PLwD). Extensive databases, including ACM Digital Library,
\end{abstract}

Cochrane Central Register of Controlled Trials, Compendex, Embase, Inspec, Ovid MEDLINE(R) Daily, Proquest Dissertations and These Global, PsycINFO, and Scopus, have been searched. All searches are from the inception of the databases. All peer-reviewed studies and articles written in the English language are included. Two reviewers will independently screen and select the studies with the involvement of a third reviewer for disagreements. Data will be abstracted using a custom data extraction form that is made based on the research questions. Critical Appraisal Skills Programme (CASP) checklists will be used to assess the study quality.

As the first review of its kind, the findings from this review will provide valuable insights related to the needs of the dementia care apps for both PLwD and their family caregivers. The review will be relevant to health providers who are interested in using technologies to promote the independence of PLWD and reduce the stress experienced from caregivers of PLwD. The review will also serve as a guide to app developers and researchers to design usable and acceptable apps. In addition, the review will provide critical knowledge of the privacy and security features of the app to reveal the valid concerns from the end users and thus help with the uptake and adoption of the dementia care apps.

(c) 2021 The Author(s)

Published by S. Karger AG, Basel karger@karger.com www.karger.com/ger

Karger $\stackrel{\text { ' }}{5}$

GOPEN ACCESS
(C) 2021 The Author(s)

Published by S. Karger AG, Basel

This is an Open Access article licensed under the Creative Commons Attribution-NonCommercial-4.0 International License (CC BY-NC) (http://www.karger.com/Services/OpenAccessLicense), applicable to the online version of the article only. Usage and distribution for commercial purposes requires written permission.
Bing Ye

Department of Occupational Science and Occupational Therapy, University of Toronto 160-500 University Ave.

Toronto, ON M5G 1V7 (Canada)

bing.ye@utoronto.ca 


\section{Introduction}

Dementia is a progressive neurodegenerative condition that can impair memory, thinking, language, orientation, behavior, and the capacity to perform daily activities [1]. Age is a major risk factor for developing dementia $[1,2]$. With the globally aging population, the chances of developing dementia will increase dramatically as a person grows older [2]. It is estimated that people living with dementia (PLwD) will reach 82 million in 2030 and 152 in 2050 worldwide [1].

PLwD primarily receive care from their family members, friends, or other unpaid caregivers in their home [3]. Several goals in dementia care have been identified, such as: optimizing the quality of life (QOL) for PLwD; providing adequate caregivers' support and guidance in providing care (i.e., behavioral symptoms managements and caregiving skills); improving community awareness in accessing dementia resources (i.e., dementia education, and support and services); the ability to live at home; and reducing psychotropic drug use for $\operatorname{PLwD}[4,5]$. Additionally, core components of the QOL for PLwD include participating in meaningful activities, enhancing feelings of safety, maintaining physical strength and mobility, retaining the ability to perform activities of daily living (ADL), and optimizing the cognitive functioning [4-7].

It is well documented that caring for a person with dementia can be overwhelming. Family and unpaid caregivers of PLwD provide significantly more hours of care, which has led to tremendous burden and stress for these informal caregivers $[1,6,8]$. According to Alzheimer's Association (2020), 16.3 million family and unpaid caregivers spent approximately 18.6 million hours caring for PLwD in 2019, resulting in an average of 21.9 hours of care provided by each caregiver per week [6]. As dementia progresses and care needs increase, $\mathrm{PLwD}$ may require regular in-home care up to 24-hour supervision [6]. Higher levels of caregivers' distress increase the likelihood of institutionalization of PLwD, which further increases the dementia care costs to the family $[9,10]$. Institutionalization is counter to the wishes of most of the older adults, who wish to stay and age at home $[11,12]$. Therefore, there is an urgent need for a solution that can support caregivers looking after PLwD to maintain the QOL and support healthy aging.

Support provided via mobile devices is an obvious option as mobile devices, including smartphones and tablet computers, have been widely adopted and are commonplace in most households around the world $[13,14]$. Today, mobile device users are not only young adults, but also include older adults as frequent users [14-16]. According to a report from Livingston (2019), the screen time among American older adults at 65 and older has been increased. There was around $60 \%$ of an increase in American older adults using internet between 2000 and 2019 (i.e., increase from $14 \%$ in 2000 to $73 \%$ in 2019) [16]. Previous studies have documented that smartphone users not only use mobile phones for basic internet browsing, texting, or calling, but also use it to download mobile apps $^{1}$ to access health-related information or to monitor aspects of personal health [14, 17]. Despite this increased usage, older adults have expressed concerns about security and privacy in relation to technology $[18,19]$.

With the prevalent use of the mobile devices, apps have been developed specifically for PLwD as users, such as apps to support ADL, memory, and safety [20,21]. Studies have shown that mobile assistive apps have the potential to enhance the QOL of PLwD, by helping them to complete tasks and promoting their independence $[20$, 22-25]. In turn, these apps can relieve caregivers' stress and improve their QOL as well [26].

Since the care and the QOL of PLwD is intimately intertwined with the QOL of their family caregivers, apps developed targeting dementia caregivers as users are also vital to strengthening dementia care in communities. However, apps that support these caregivers in providing care are limited [27]. Despite the capacity for apps to assist in dementia care and their potential in reducing the care cost, there is a dearth of knowledge in understanding the needs of PLwD and their caregivers as they relate to these care apps $[27,28]$. Therefore, it is important to conduct a comprehensive review that assesses available dementia care apps, and how they address the needs of PLwD and their informal caregivers. As such, the major research questions of this systematic review are to identify:

1. What types and features (i.e., professional referral) of apps have been created in supporting dementia care (i.e., QOL, dementia education, support and services, and performing $\mathrm{ADL}$ ) for PLwD and their informal caregivers?

2. What Operating System (O.S.) and devices are associated with these dementia care apps?

3. What are the privacy and safety features of the apps, from perspectives of both end users (i.e., concerns of being hacked) and developers/researchers (i.e., apps with security prompts built in, privacy policy, or encrypted data)?

\footnotetext{
Mobile app or mobile application: It is a software program that is designed specifically for use on smartphones or tablets.
} 
4. Which apps are commercially available?

5. What methodologies have been used for usability evaluation?

6. What are the results obtained from the usability evaluation of the apps?

\section{Methods}

\section{Study Method}

The systematic review will follow the guidelines of Preferred Reporting Items for Systematic Reviews and Meta-Analyses (PRISMA) [29]. This systematic review protocol has been registered to the PROSPERO International Prospective Register of Systematic Reviews (Registration ID: CRD42020216141).

\section{Search Strategy}

Electronic Databases

The search strategy has been developed in consultation with, and conducted by, an information specialist. An extensive database was searched for relevant articles including:

- ACM Digital Library

- Cochrane Central Register of Controlled Trials

- Compendex

- Embase

- Inspec

- Ovid MEDLINE(R) Daily

- Proquest Dissertations and These Global

- PsycINFO

- Scopus

The search strategy included both text and index terms related to the following two areas: (1) dementia or Alzheimer's and (2) mobile apps. Please see the online supplementary document (see www.karger.com/doi/10.1159/000514838 for all online supplementary material) of the full list of the search strategy.

\section{Manual Searches}

The reference list of the included studies in this systematic review, relevant systematic reviews, and other relevant publications will be manually searched to identify additional potentially relevant studies.

\section{Inclusion and Exclusion Criteria}

The search was limited to human participants and to articles written in the English language only, and the search started from the inception of the databases. Articles that meet the following criteria will be included:

- Study participants: the study includes either PLwD living in the community, or informal caregivers of PLwD living in the community, or both.

- Studies focusing on dementia care apps on smartphone, handheld laptop/computer, or tablet.

- Studies that include and evaluate the usability of the app by end users.

Articles will be excluded if any of the following criteria is met:

- The study that is focusing on a non-smartphone, such as a phone that is not able to download an app, or a phone that does not have a touchscreen function.
- The study participants are not human.

- The study participants are healthy older adults, PLwD living in a nursing home, or long-term care.

- The study is focused on apps on mental health, physical health, and well-being broadly and are not dementia specific.

- The study is focused on apps developed for therapists, nurses, clinicians, doctors, and/or professional caregivers.

- The study is focused on apps that are not related to dementia care at home, such as cognitive assessment or dementia diagnosis.

- The study is only describing the concept of the app development.

- The study is only describing how the app is designed.

- The study is only describing an app itself.

- Review articles, such as the systematic review and literature review.

- Studies that are not peer-reviewed.

- Presentations, symposiums, supplements, commentaries, abstracts, editorials, and posters.

\section{Study Selection}

Endnote will be used to import the references from the database results. Duplicates will be removed by one reviewer (B.Y.) from the endnote. The references after the duplicates have been removed will then be exported to COVIDENCE. There will be two stages of screening for study selection: title and abstract screening, followed by full-text screening. Two reviewers (B.Y. and T.V.H.) will independently conduct the title and abstract screening by reading the titles and abstracts and determine their eligibility based on the inclusion and exclusion criteria. Any references that are in disagreement between the two reviewers will be recorded and marked. These references will first be discussed by the two reviewers for consensus. Any references with disagreement after discussion will be resolved by a third reviewer (C.C.). The remaining references will then undergo full-text screening. Only one reviewer (B.Y.) will review these references for the final inclusion of the studies based on the same inclusion and exclusion criteria mentioned above. The study selection process will follow a PRISMA study flow diagram.

\section{Data Extraction}

The final included studies after the full-text screening will be abstracted by a reviewer (B.Y.) on a custom data extraction form. The custom data extraction form will be made based on the research questions and recommended best practices for reviewing healthcare technology [30]. This form will be piloted over a random sample of the included studies, reviewed for coding consistency of extracted data, and then refined to best meet the goals of this study [30]. The extracted data will be independently verified by a second reviewer (T.V.H.). Any discrepancies will be resolved by a third reviewer (C.C.). The information that will be extracted includes the following:

- Publication details: authors, publication year, country of origin, funding source, and study title.

- Study design, such as case study, focus group, interview study, observational study, or cohort study.

- Participant information including types of participants (i.e., informal caregivers and/or PLwD), sample size, participant's age and gender, stages of dementia (i.e., mild or moderate), participants with previous experience on technology/computer or touch screen/mobile phones. 
- Aim of the study.

- Information of the dementia care app: name of the app if applicable, language that is offered by the app, O.S., types of device used (i.e., mobile phones, or tablet), app types (i.e., reminders, navigations, and providing dementia care information), apps support to caregivers, PLwD or both, and commercialization of the app, professional referral as a feature, security, and privacy features of the app, the kind of data being entered into the app by the user and data being collected automatically such as GPS.

- Methodologies and scales for app usability evaluation.

- Results: results of the usability evaluation and overall conclusion of the study.

\section{Quality Assessment}

We expect there may be various types of study design. Therefore, we will use the Critical Appraisal Skills Programme (CASP) checklists [31]. There are different CASP checklists corresponding to different study designs. For example, all qualitative studies will be assessed by CASP qualitative studies checklist. RCT studies will be assessed by CASP RCT checklist. The quality assessment of included studies will be completed by two reviewers (B.Y. and T.V.H) independently. If there is disagreement on particular studies, it will be resolved by discussion between the two reviewers, or with the consultation of the third reviewer (C.C.), if necessary.

\section{Analyses}

A summary table of the study characteristics will be created for descriptive information including the authors, publication year, participant types, sample size, stages of dementia, test period, and study design.

A narrative synthesis of the findings from the data extraction table of the included studies will be conducted. The findings will be organized based on the 6 main research questions. This will include:

1. What types and features of apps (i.e., professional referral) have been created in supporting dementia care for PLwD and their informal caregivers, respectively including the goals of the apps, the data they collect, and features of the app like clinician referrals?

2. What O.S. and devices are associated with these dementia care apps?

3. What are the privacy and safety feature of the app, from perspectives of both end users (i.e., concerns of being hacked) and developers/researchers (i.e., apps with security prompts built in, privacy policy, or encrypted data etc.)?

4. Which apps are commercially available?

5. What methodologies have been used for usability evaluation?

6. What are the results obtained from the usability evaluation of the apps?

An inductive content analysis approach [32] will be applied. Specifically, texts related to the results from the research questions will be coded and labeled for concepts [33]. Repeated or similar codes will be grouped into categories [33]. The lists of categories will then be grouped into broader categories $[34,35]$. The new lists of categories will then be grouped to more general categories. This process will be repeated until exhaustion is reached among categories [35]. NVivo will be used for this qualitative data analysis.

As there may be heterogeneity of outcome measures in the included studies, a meta-analysis may not be suitable in this review.
However, where appropriate, results will be pooled using a random effect meta-analysis [36]. To be specific, data will be pooled when studies are homogeneous based on participant cohorts (i.e., PLwD), methodological approaches (i.e., longitudinal study), type of the app in dementia care (i.e., navigation), and outcome measure, such as the app performance. Study heterogeneity will be measured by the $I^{2}$ statistics, which defines as "the percentage of total variation across studies that is due to heterogeneity rather than chance" [37]. $I^{2}$ ranges between 0 and $100 \%$. There is tentatively three benchmarks to describe the heterogeneity for $I^{2}$ values. Heterogeneity is considered as low, moderate, and high with values of $25 \%, 50 \%$, and $75 \%$, respectively [37].

\section{Patient and Public Involvement}

As it is a systematic review, we do not expect patient and the public to be involved.

\section{Discussion}

As the first of its kind, this systematic review will present a comprehensive review on the types and features of available apps in dementia care for both PLwD and their informal caregivers. This review will be relevant for PLwD and their family members and/or friends who provide care to them. The faster growth of mobile device and its widespread use, made it the inseparable part of people's daily life. Mobile apps have great potential in non-pharmacological intervention for dementia care. These dementia care apps also have a greater potential in improving the QOL of both PLwD and their informal caregivers, and reduce caregivers' burden. As such, this review will be of great relevance for healthcare providers and researchers who are interested in developing interventions and technologies to promote the independence of PLwD and reduce the stress experienced from caregivers of PLwD.

A recent study indicated that security and privacy, including fear of hacking was among the top reasons, why older adults are hesitant to use technology [18]. There are also commercial and potential financial interests in developing a tool for this population, in particular the sharing of private information to third parties [38]. For this reason, it is important this review analyzes the types of data being collected, the security and privacy features of the apps, and where the data are being stored. There is a knowledge gap in this area when it pertains to apps aimed at older adults, and this review can provide critical knowledge that may quell the valid concerns of users.

In addition, this review will provide valuable insights regarding the needs of the apps in supporting dementia care from the perspectives of both PLwD and their infor- 
mal caregivers. The difficulties a user experiences in interacting with an app could result in its rejection. Understanding the users' needs and designing a usable app that can be used by both PLwD and their caregivers will increase the adoption of the app. This review will serve as a guide to app developers and researchers, who are exploring and developing new applications for PLwD, and their family members and friends.

\section{Acknowledgements}

We would like to thank our Information Specialist, Ms. Jessica Babineau from the University Health Network, who has contributed her knowledge conducting the extensive database search for this systematic review.

\section{Conflict of Interest Statement}

The authors have no conflicts of interest to declare.

\section{Funding Sources}

This study did not receive any funding.

\section{Author Contributions}

B.Y. designed and developed the systematic review. B.Y. drafted the protocol. All authors contributed in review concept. All authors provided critical revisions for important intellectual content. All authors approved the final version of the manuscript. All authors agreed to be accountable for all aspects of the work.

\section{References}

1 World Health Organization. Dementia 2020. Available from: https://www.who.int/news$\mathrm{room} /$ fact-sheets/detail/dementia.

2 Alzheimer Society Canada. Risk factors 2020. Available from: https://alzheimer.ca/en/ Home/About-dementia/Alzheimer-s-disease/Risk-factors.

3 Centers for Disease Control and Prevention. Caregiving for family and friends: a Public Health Issue. 2019.

4 Crisis Prevention Institute. How to establish dementia care best practices and why they matter 2020. Available from: https://www.crisisprevention.com/Blog/Dementia-CareBest-Practices.

5 Jennings LA, Palimaru A, Corona MG, Cagigas XE, Ramirez KD, Zhao T, et al. Patient and caregiver goals for dementia care. Qual Life Res. 2017;26(3):685-93.

6 Alzheimer's Association. 2020 Alzheimer's Disease Facts and Figures. 2020. p. 391-60.

7 Logsdon RG, McCurry SM, Teri L. EvidenceBased Interventions to Improve Quality of Life for Individuals with Dementia. Alzheimers care today. 2007;8(4):309-18.

8 Kasper J, Freedman V, Spillman B. Disability and care needs of older Americans by dementia status: an analysis of the 2011 national health and aging trends study. Washington, DC: US Department of Health and Human Services; 2014.

9 de Vugt ME, Stevens F, Aalten P, Lousberg R, Jaspers N, Verhey FR. A prospective study of the effects of behavioral symptoms on the institutionalization of patients with dementia. Int Psychogeriatr. 2005;17(4):577.

10 Maust DT, Kales HC, McCammon RJ, Blow FC, Leggett A, Langa KM. Distress associated with dementia-related psychosis and agitation in relation to healthcare utilization and costs. Am J Geriatr Psychiatry. 2017;25(10):1074-82.
11 Zank S, Schacke C. Evaluation of geriatric day care units: effects on patients and caregivers. J Gerontol B Psychol Sci Soc Sci. 2002;57(4): P348-57.

12 National Institute on Aging. Aging in place: growing older at home. National Institute on Aging. 2017 [cited 2020 Oct 23]. Available from: https://www.nia.nih.gov/health/agingplace-growing-older-home.

13 Pew Research Center. Mobile Fact Sheet. Washington, DC 20036, USA; 2019.

14 Paré G, Bourget C. Diffusion of smart devices for health in Canada. Montreal, Quebec, Canada: CEFRIO; 2017.

15 Anderson M, Perrin A. Tech adoption climbs among older adults. Washington, DC 20036, USA; 2017.

16 Livingston G. Americans 60 and older are spending more time in front of their screens than a decade ago. Washington, DC 20036, USA; 2019.

17 Smith A. Smartphone use in 2015. USA: Pew Research Center; 2015.

18 Quan Haase A, Ho D. Online privacy concerns and privacy protection strategies among older adults in East York, Canada. JASIST. 2020.

19 Frik A, Nurgalieva L, Bernd J, Lee J, Schaub F, Egelman S. Privacy and security threat models and mitigation strategies of older adults. Fifteenth symposium on usable privacy and security ([SOUPS] 2019); 2019.

20 Klimova B, Bouckova Z, Toman J. Mobile phone apps as support tools for people with dementia. Advanced Multimedia and Ubiquitous Engineering: Springer; 2018. p. 7-12.

21 Armstrong N, Nugent C, Moore G, Finlay D. Developing smartphone applications for people with Alzheimer's disease. Proceedings of the 10th IEEE International Conference on Information Technology and Applications in Biomedicine. IEEE; 2010.
22 Lanza C, Knörzer O, Weber M, Riepe MW. Autonomous spatial orientation in patients with mild to moderate Alzheimer's disease by using mobile assistive devices: a pilot study. J Alzheimers Dis. 2014;42(3):879-84.

23 Leng FY, Yeo D, George S, Barr C. Comparison of iPad applications with traditional activities using person-centred care approach: impact on well-being for persons with dementia. Dementia. 2014;13(2):265-73.

24 Jönsson K-E, Ornstein K, Christensen J, Eriksson J. A reminder system for independence in dementia care: a case study in an assisted living facility. Proceedings of the 12th ACM international conference on pervasive technologies related to assistive environments; 2019.

25 Bier N, Brambati S, Macoir J, Paquette G, Schmitz X, Belleville S, et al. Relying on procedural memory to enhance independence in daily living activities: Smartphone use in a case of semantic dementia. Neuropsychol Rehabil. 2015;25(6):913-35.

26 Chauvet R, Rasche P, Berti Z, Wille M, Barton L, Schäfer K, et al. Satisfying product features of a dementia care support smartphone app and potential users' willingness to pay: webbased survey among older adults. International conference on applied human factors and ergonomics. Springer; 2019.

27 Brown EL, Ruggiano N, Li J, Clarke PJ, Kay ES, Hristidis V. Smartphone-based health technologies for dementia care: opportunities, challenges, and current practices. J Appl Gerontol. 2019;38(1):73-91.

28 Guo Y, Yang F, Hu F, Li W, Ruggiano N, Lee HY. Existing mobile phone apps for self-care management of people with alzheimer disease and related dementias: systematic analysis. JMIR Aging. 2020;3(1):e15290. 
29 Moher D, Liberati A, Tetzlaff J, Altman DG, Group P. Preferred reporting items for systematic reviews and meta-analyses: the PRISMA statement. PLoS Med. 2009;6(7): e1000097.

30 Büchter RB, Weise A, Pieper D. Development, testing and use of data extraction forms in systematic reviews: a review of methodological guidance. BMC Med Res Methodol. 2020;20(1):259.

31 Critical Appraisal Skills Programme. CASP Checklists 2019 [cited 2020]. Available from: https: //casp-uk.net/\#!casp-tools-checklists/ c18f8.
32 Elo S, Kyngäs H. The qualitative content analysis process. J Adv Nurs. 2008;62(1):107-15.

33 Khandkar SH. Open coding. University of Calgary; 2009. p. 23.

34 Burnard P. A method of analysing interview transcripts in qualitative research. Nurse Educ Today. 1991;11(6):461-6.

35 Thomas DR. A general inductive approach for analyzing qualitative evaluation data. Am J Eval. 2006;27(2):237-46.
36 Borenstein M, Hedges LV, Higgins JP, Rothstein HR. Introduction to meta-analysis. John Wiley \& Sons; 2011.

37 Higgins JP, Thompson SG, Deeks JJ, Altman DG. Measuring inconsistency in meta-analyses. BMJ. 2003;327(7414):557-60.

38 Grundy Q, Chiu K, Held F, Continella A, Bero L, Holz R. Data sharing practices of medicines related apps and the mobile ecosystem: traffic, content, and network analysis. BMJ. 2019; 364:1920. 